\title{
Die Bedeutung der Radioaktivität in der Wasserversorgung und Abwasserreinigung
}

\author{
Von W. STUMM
}

(Mitteilung Nr. 93 der Eidgenössischen Anstalt für Wasserversorgung, Abwasserreinigung und Gewässerschutz an der Eidg. Technischen Hochschule in Zürich)

Manuskript eingegangen am I6. Juni 1955

\section{EINLEITUNG}

Innerhalb der letzten zehn Jahre sind vornehmlich in der amerikanischen und englischen Fachliteratur der Wasserversorgung und Abwasserreinigung zahlreiche Arbeiten uiber Probleme der Verunreinigung der Gewässer durch radioaktive Stoffe und über die Beseitigung radioaktiver Materialien erschienen. Die Entfernung von radioaktiven Verunreinigungen aus dem Wasser ist heute eine wichtige und aktuelle Aufgabe der Abwasserreinigung geworden.

Auch in der Schweiz müssen wir damit rechnen, dass in näherer Zukunft solche Probleme zum Aufgabenbereich der Wasser- und Abwasserfachleute gehören werden. Es erscheint uns deshalb angemessen, die wichtigsten Gesichtspunkte zusammenzustellen.

\section{Herkunft radioaktiver Verunreinigungen}

Radioaktive Verunreinigungen können verschiedensten Ursprungs sein:

r. Kernreaktoren und Isotopenaufbereitungsanstalten.

2. Anwendung von Radioisotopen in Industrie, Forschung und Medizin.

3. Atomische Explosionen.

Kernreaktoren sind die grössten potentiellen Quellen radioaktiver Verunreinigung. So wird zum Beispiel in Hanford (USA.) zur Kuihlung der Uranbrenner dem Columbia-Fluss eine Wassermenge von rund $500 \cdot 10^{6} 1$ pro Tag entnommen. Nach einmaliger Zirkulation enthält dieses Wasser eine Aktivität, die eine direkte Wiederabgabe des verbrauchten Kühlwassers 
an den Fluss nicht mehr ratsam erscheinen lässt [46]. Es ist allerdings heute möglich, Reaktoren zu bauen, in denen die Kühlung der Uranbrenner so vorgenommen werden kann, dass kein radioaktives $A$ bwasser entsteht. In der Schweiz wird in den nächsten Jahren in Würenlingen ein Kernreaktor für Energieerzeugungszwecke gebaut. Dieses Jahr wurde ein kleiner Forschungs- und Materialprüfungsreaktor von den Vereinigten Staaten angekauft. Ebenfalls dieses Jahr wurde in Genf der Grundstein für das Laboratorium des Conseil Européen de la Recherche Nucléaire (C.E.R.N.) gelegt. In diesem Forschungsinstitut wird ein Cyclotron und ein Proton-Synchroton gebaut werden. Für den Reaktor in Würenlingen wurde versichert, dass dieser keine unmittelbare Gefahr für eine radioaktive Verunreinigung darstellen werde. Beim Betrieb eines Reaktors entstehen hingegen radioaktive Beiprodukte, die auf möglichst unschädliche Art aufbereitet oder beseitigt werden müssen.

Ferner muss angenommen werden, dass durch die Erstellung von Kernreaktoren die Verwendung von radioaktiven Stoffen in Industrie, Forscbung. und Medizin zunehmen wird. Bis heute müssen die Radioisotope aus dem Ausland bezogen werden. Sie waren deshalb auch recht kostspielig. Die Anwendung radioaktiver Isotope wird bei uns, sobald diese relativ einfach und preiswert in der Schweiz erhältlich sind, eine wesentliche Verbreiterung erfahren. Radioaktive Stoffe sind wertvolle Werkzeuge für die Lösung von technischen Problemen und Forschungsaufgaben. So können in der Chemie und Biologie die Unterscheidbarkeit von isotopen Atomarten und/ oder ihre leichte und empfindliche Nachweisbarkeit ausgenützt werden (Tracer-Methode [6], [8], [9]). Die Chemie hat durch die Verwendung von Leitisotopen weitgehende Möglichkeiten zur Analyse von. Reaktionen. In der Biologie konnten mit Hilfe radioaktiver Indikatoren viele Probleme der Stoffwechselanalyse gelöst werden. In den Vereinigten Staaten ist man darangegangen, Lebensmittel mit Hilfe von Strahlen zu desinfizieren, und Grossanlagen zur Strahlungsentkeimung von Kartoffeln sind in Betrieb. Die medizinischen Anwendungen von radioaktiven Stoffen in Diagnostik und Therapie sind zahlreich und werden immer mehr zunehmen. (Das Kantonsspital Zürich hat letztes Jahr für Fr. iı ooo.- Radioisotope aus England bezogen.)

Es ist auch auf die Möglichkeit einer Beeinflussung der schweizerischen Oberflächengewässer durch atomische Explosionen [27] hinzuweisen. In diesem Zusammenhang mag erwähnt werden, dass wenige Tage nach atomischen Versuchsexplosionen in Nevada im Regenwasser und in den Oberflächengewässern von Massachusetts (Distanz Nevada-Massachusetts 
rund $5000 \mathrm{~km}$ ), wenn auch nicht gefährliche, so doch einwandfrei feststellbare Aktivitäten gemessen werden konnten [29].

Der Schutz der Gewässer (Oberflächen- und Grundwasser) vor radioaktiver Verunreinigung ist in erster Linie im Hinblick auf die Trinkwasserversorgung wichtig. Andererseits wurde nachgewiesen, dass gewisse Wasserorganismen radioaktive Isotope in grosser Menge speichern können. Daraus ergibt sich für den Menschen die Möglichkeit der indirekten Gefährdung (zum Beispiel der Genuss von radioaktiv gewordenen Fischen [67]). Ferner dürfen allgemein biologische (biohygienische) Gesichtspunkte nicht ausser acht gelassen werden.

\section{PHYSIOLOGISCHE GESICHTSPUNKTE}

\section{ZUR FESTLEGUNG VON ZULÄSSIGEN STRAHLENDOSEN}

Ionisierende Strahlung kann Schädigungen des tierischen oder menschlichen Organismus bewirken. Kleinste Bezirke in der Zelle können durch wenige Ionisationen oder sogar durch eine einzelne Ionisation verändert werden. Schädigungssymptome beim Menschen sind bei geringer Bestrahlung Übelkeit und Erythema, bei stärkerer Bestrahlung blutige Diarrhöe, Fieber, Haarausfall, Sterilität und Geschwürbildung. Häufig tritt Anämie auf, die in schweren Fällen zum Tode führen kann. Eine chronische Schädigung äussert sich meist in einer Verminderung der weissen Blutkörperchen. Ferner werden genetische Mutationen induziert. Diese Strahlungswirkung kann von aussen her erfolgen, wenn sich der Organismus im Strahlungsbereich radioaktiver Substanzen befindet, oder von innen her durch Aufnahme von Radioisotopen in den Stoffwechsel. Einige Radioisotope können auch unabhängig von der Aktivität durch die chemische Natur den Organismus schädigen.

Es ist ausserordentlich schwierig, geeignete allgemeingültige und vergleichbare Masseinheiten für die Strahlendosis aufzustellen (gebräuchliche Strahlungseinheiten sind im Anhang zusammengestellt). Es bestehen auch Anhaltspunkte dafür, dass verschiedene Personen auf Strahlung verschieden empfindlich reagieren [Io]. Die Strahlendosis setzt sich als Produkt von Strahlungsintensität und Bestrahlungszeit zusammen. Innerhalb gewisser Grenzen wird der Schädigungseffekt durch die Geschwindigkeit, mit welcher Strahlung vom Gewebe absorbiert wird, nicht beeinflusst (zum Beispiel hat eine ganztägige Exposition von 4,17 mr [Milliröntgen] pro Stunde denselben Effekt wie eine Bestrahlung von $400 \mathrm{mr}$ während 
I5 min). Andererseits kann eine Dosis von 36,5 r [Röntgen], die nur an einem Tag jedes Jahres absorbiert wird, grösseren Schaden anrichten als eine jahrelange tägliche Exposition von $0, \mathrm{I} r$. Es ist in beschränktem Masse möglich, sich von Strahlenschäden zu erholen.

Im Hinblick auf genetische Effekte scheint es hingegen festzustehen, dass sich die Dosisleistungen von Gammastrahlen (oder Röntgenstrahlen) streng kumulativ verhalten. Die Anzahl der durch Strahlen induzierten Mutationen in den Zellen ist unabhängig davon, ob eine bestimmte Strahlendosis in einer einzigen Bestrahlung oder verteilt über mehrere Jahre empfangen wird. Wenn man eine Bevölkerungsgruppe als Ganzes in Betracht zieht, so ist die Anzahl induzierter Mutationen unabhängig davon, ob die Keimzellen von too Personen eine Dosis von je Ioo r oder von Ioo 000 Personen von je 0, I $\mathrm{r}$ in irgendeiner Zeitspanne erhalten. Die durchschnittliche Wahrscheinlichkeit einer Mutation wird bei einer Strahlendosis von $\mathrm{Ir}$ auf $\mathrm{I} \%$ für jedes individuelle Gen geschätzt [20]. So würde zum Beispiel nach Schätzungen von C. STERN [20] eine Person, die während zehn Jahren pro Arbeitstag einer Strahlung von $0, \mathrm{I} r$ (der früher üblichen Toleranzdosis) ausgesetzt wäre, eine Dosis von etwa $300 \mathrm{r}$ akkumulieren, welche die Mutationsrate in ihren Keimzellen gegenüber der natürlichen Rate um $\mathrm{I} 50 \%$ erhöhen könnte. Mutationen haben in der Mehrzahl negativen Selektionswert.

Vor allem vom genetischen Gesichtspunkt aus muss deshalb die Exposition gegenüber allen Arten ionisierender Strahlung auf das kleinstmöglichste Mass reduziert werden. Andererseits dürfen die durch sehr geringe Strahlenintensitäten bewirkten Gefahren auch nicht überschätzt werden. Es ist zu berücksichtigen, dass wir alle dauernd in einem geringen Masse ionisierender Strahlung ausgesetzt sind. Diese stammt zum Teil von natürlicher Radioaktivität. Die Konzentration an Radon in der Luft beträgt etwa $10^{-13} \mathrm{c} / \mathrm{cm}^{3}$ ( $\mathrm{c}=$ Curie), im Wasser etwa $10^{-15} \mathrm{c} / \mathrm{cm}^{3}[3]$, [32]. Die davon herrührende Strahlendosis wird durch die kosmische Strahlung noch erhöht, so dass wir einer Strahlung von $\mathrm{I}-5 \cdot \mathrm{IO}^{-5} \mathrm{r}$ pro Stunde ausgesetzt sind [16].

Heute fehlen noch genügende experimentelle Grundlagen für die Festlegung einer exakten Toleranzdosis. Zahlreiche Forschungsarbeiten über die physiologische Wirkung ionisierender Strahlung sind in biologischen und medizinischen Instituten im Gange. Die National Academy of Science (USA.) hat im Mai I955 angekündigt, dass sie mit Hilfe der Rockefeller Foundation derartige Untersuchungen auf breiter Grundlage durchführen werde. Es ist nicht möglich, sowohl wegen der Mannigfaltigkeit der Re- 
aktionen der verschiedenen Radioisotope im Stoffwechsel als auch wegen der Verschiedenheit der Strahlenarten die Strahlenexposition des Organismus durch einen einzigen Wert zu definieren. In den angelsächsischen Ländern gelten die in Tabelle I zusammengestellten Werte für die höchstzulässige Strahlendosis (Toleranzdosis). Die Toleranzdosen, die von der International Commission on Radiological Protection [12] aufgestellt worden sind, enthalten Zahlenwerte, die nicht wesentlich davon abweichen.

'Tabelle 1

Zulässige Strablendosis in rep ${ }^{1}$ ) pro Woche [9a]

\begin{tabular}{|c|c|c|c|}
\hline \multirow{2}{*}{ Typus der Strahlung } & $\begin{array}{c}\text { Innerhalb } \\
\text { des } \\
\text { Körpers }\end{array}$ & $\begin{array}{c}\text { In der äussersten Schicht } \\
\text { der Epidermis }\end{array}$ \\
\cline { 3 - 4 } & a) Exposition des \\
ganzen Körpers & $\begin{array}{c}\text { b) Exposition } \\
\text { der Hände }\end{array}$ \\
\hline $\begin{array}{l}\text { Gamma-(Röntgen-) strahlen } \\
\text { und Betastrahlen . . . }\end{array}$ & 0,3 & 0,5 & 1,5 \\
Alphastrahlen . . . . & 0,015 & 0,025 & 0,075 \\
\hline 1) Definition des rep siehe Anhang. & & & \\
\hline
\end{tabular}

Von den zulässigen Strahlendosen können die zulässigen Mengen der verschiedenen radioaktiven Substanzen, die im Organismus deponiert werden dïrfen, abgeleitet werden, sofern die charakteristischen Merkmale der Absorption im Organismus bekannt sind. Es ist notwendig zu wissen, in welchem Organ das entsprechende Radioisotop akkumuliert und mit welcher Geschwindigkeit es wieder ausgeschieden wird. Die Wirksamkeit eines bestimmten Radioisotops im Körper ergibt sich demnach durch die Zerfallsgeschwindigkeit des Isotops einerseits und durch die Eliminationsgeschwindigkeit des Isotops im Organismus andererseits. Die Zerfallsgeschwindigkeit kann durch die entsprechende radioaktive Halbwertszeit $\left(T_{r}\right)$, die Ausscheidungsgeschwindigkeit durch die sogenannte biologische Halbwertszeit $\left(T_{b}\right)$ charakterisiert werden. Die effektive Halbwertszeit $\left(T_{e}\right)$ ergibt sich demnach aus folgender Bezichung:

$$
T_{e}=\frac{T_{r} T_{b}}{T_{r}+T_{b}}
$$

In Tabelle 2 sind für einige Radioisotope die entsprechenden Halbwertszeiten aufgeführt. In Tabelle 3 sind die in den Vereinigten Staaten gültigen [17] und die von der International Commission on Radiological Protection 
Tabelle 2

Radioaktive und biologische Halbmertszeiten einiger Radioisotope [16]

\begin{tabular}{|c|c|c|c|c|c|}
\hline \multirow[b]{2}{*}{ Isotop } & \multirow[b]{2}{*}{ Strahlungsart } & \multirow{2}{*}{$\begin{array}{l}\text { Organ, in dem } \\
\text { das Isotop } \\
\text { deponiert } \\
\text { wird }\end{array}$} & \multicolumn{3}{|c|}{ Halbwertszeit } \\
\hline & & & $\begin{array}{c}\text { radioalktive } \\
\left(T_{p}\right)\end{array}$ & $\begin{array}{c}\text { biolo- } \\
\text { gische } \\
\left(T_{b}\right) \text { Tage }\end{array}$ & $\begin{array}{c}\text { effek- } \\
\text { tive } \\
\left(T_{e}\right) \text { Tage }\end{array}$ \\
\hline Kohlenstoff 14 & Beta & Ganzer Körner & & & \\
\hline Natrium 24 & Beta,Gamma & $\begin{array}{l}\text { Ganzer Korper } \\
\text { Gärer Ker }\end{array}$ & $\begin{array}{l}3000 \mathrm{Jahre} \\
14,8 \mathrm{~h}\end{array}$ & $\begin{array}{l}180 \\
29\end{array}$ & $\begin{array}{l}180 \\
0.61\end{array}$ \\
\hline Phosphor 32 & Beta & \{a) Knochen $\}$ & 14.3 Tage & \{172 & 13,2 \\
\hline Calcium 45 & Beta & $\begin{array}{l}\text { (b) Muskeln } 1 \\
\text { Knochen }\end{array}$ & 152 Tage & $\begin{array}{l}395 \\
18000\end{array}$ & 13,8 \\
\hline Kobalt 60 & Beta, Gamma & Leber & 5,3 Jahre & 8,4 & $\begin{array}{l}151 \\
8,4\end{array}$ \\
\hline $\begin{array}{l}\text { Strontium } 89 \\
\text { Strontium } 90\end{array}$ & Beta & Knochen & 54 Tage & 3900 & 52 \\
\hline $\left.\begin{array}{l}\text { Strontium 90 } \\
+ \text { Yttrium } 90\end{array}\right\}$ & Beta & Knochen & 24 Jahre (Sr) & 3900 & 2700 \\
\hline Jod 131 & Beta, Gamma & Schilddrüse & 8 Tage & 180 & 7,7 \\
\hline $\begin{array}{l}\text { Gold } 198 \\
\text { Radon } 222\end{array}$ & Beta, Gramma & Nieren & 2,7 Tage & 50 & 2,6 \\
\hline Radium 226 & $\begin{array}{l}\text { Alpha } \\
\text { Alpha, Gamma }\end{array}$ & $\begin{array}{l}\text { Ganzer Körper } \\
\text { Knochen }\end{array}$ & $\begin{array}{l}3,8 \text { Tage } \\
1600 \text { Iahre }\end{array}$ & $\overline{16}$ & - \\
\hline Uran 235 & Alpha, Gamma & Nieren, Lungen & $\begin{array}{l}1600 \text { Jahre } \\
9 \cdot 10^{8} \text { Jahre }\end{array}$ & $\frac{16000}{-}$ & 16000 \\
\hline Uran 238 & Alpha & $\begin{array}{l}\text { Nieren, Lungen } \\
\text { Knochen }\end{array}$ & $4,5 \cdot 10^{9}$ Jahre & 30 & 30 \\
\hline
\end{tabular}

empfohlenen Toleranzkonzentrationen einiger Radioisotope zusammengestellt. Diese Werte bewirken in keinem Teil des Organismus höhere Strahlendosen als die in Tabelle I aufgeführten. Gleichungen zur Berechnung dieser Konzentrationen sind von MORGAN [r6] entwickelt worden.

Vergleichsweise sind in Tabelle 4 die Radioaktivitätskonzentrationen (Radon) einiger Mineralquellen zusammengestellt. Die dort genannten Zahlen sollen illustrieren, dass Quellen, welche allerdings hauptsächlich für Badezwecke benützt werden, diese maximal zulässigen Konzentrationen wesentlich überschreiten können.

Die in Tabelle I und 3 aufgeführten «Toleranzwerte» dürfen nicht als endgültig betrachtet werden. Sie müssen immer wieder neuen naturwissenschaftlichen Erkenntnissen angepasst werden.

In der Schweiz werden von einer Expertenkommission Richtlinien und Verordnungen über die Beachtung von Sicherheitsmassnahmen beim Arbeiten mit ionisierender Strahlung ausgearbeitet.

\section{BESEITIGUNG RADIOAKTIVER STOFFE}

Bei der Gewinnung und Verwendung radioaktiver Isotope entstehen meistens radioaktive Abwässer und Abfallprodukte. 
XVII, 1955 Radioaktivität in der Wasserversorgung und Abwasserreinigung

Tabelle 3

Zulässige Konzentration einiger Radioisotope in Trinkmasser und in Luft [12], [17]

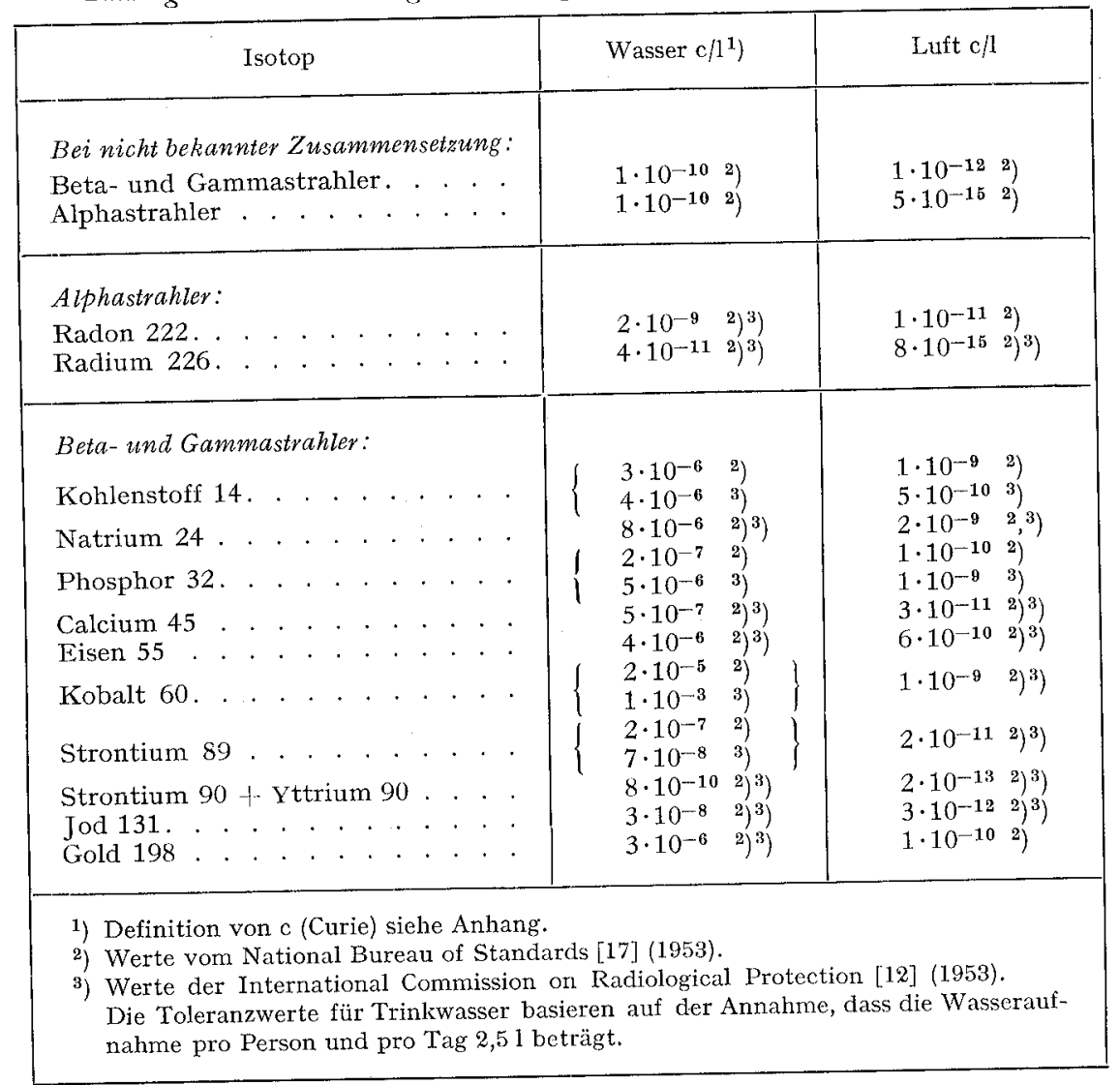

Tabelle 4

Radioaktivität einiger Mineralquellen (nacb Gübeli [66])

\begin{tabular}{|c|c|c|c|c|}
\hline Quelle & & & & Aktivität (Radon) c/l \\
\hline $\begin{array}{l}\text { Schinznach-Bad . } \\
\text { Lavey-les-Bains (bei St-Maurice, VS) } \\
\text { San Pancrazio, Stabio (TI) } \\
\text { Quellgase. }\end{array}$ & $\begin{array}{ll}\cdot & \cdot \\
\cdot & \cdot \\
\cdot & \cdot \\
. & \cdot \\
\cdot & \cdot \\
. & \cdot\end{array}$ & $\begin{array}{l}\cdot \\
\cdot \\
\cdot \\
\cdot \\
\cdot\end{array}$ & 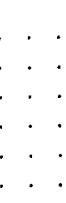 & $\begin{array}{r}9,6 \cdot 10^{-10} \\
41,8 \cdot 10^{-10} \\
16,6 \cdot 10^{-10} \\
59 \cdot 10^{-10} \\
202 \cdot 10^{-10} \\
7200 \cdot 10^{-10}\end{array}$ \\
\hline
\end{tabular}


Radioaktive Abrsässer unterscheiden sich nicht wesentlich von Abwässern, die chemische Gifte enthalten. Sie dürfen nicht unbehandelt und unkontrolliert an die Vorfluter abgegeben werden. Radioaktive Abwässer sind sowohl ihrer Menge als auch ihrer Zusammensetzung nach sehr wechselhaft. Meist ist ihr Anfall stossweise. Radioaktive Stoffe können durch chemische oder physikalische Prozesse nicht unschädlich gemacht werden, wie das bei chemischen Giften oft erfolgreich möglich ist. So kann zum Beispiel in Abwässern aus galvanischen Anstalten das ausserordentlich giftige Zyanid durch Oxydation mit Chlor in das physiologisch harmlosere Zyanat umgewandelt werden. Die Giftwirkung radioaktiven Materials hingegen wird durch keine chemische Umsetzung verändert.

Es gibt drei prinzipielle Möglichkeiten, radioaktive Abwässer unschädlich zu machen:

I. Das Abwasser wird gestapelt und so lange aufbewahrt, bis seine Aktivität durch Zerfall der Isotope genügend klein geworden ist.

2. Verdünnung.

3. Entfernung des radioaktiven Materials aus dem Abwasser.

\section{Stapelung}

Das Verfahren der Stapelung radioaktiver Wasser lässt sich vor allem dann anwenden, wenn die radioaktiven Verunreinigungen grosse Zerfallsgeschwindigkeiten aufweisen. So sinkt die Aktivität eines mit Natrium 24 verunreinigten Wassers innerhalb $\mathrm{I}_{50} \mathrm{~h}$ auf $\mathrm{O}, \mathrm{I} \%$ des ursprünglichen Wertes. Andererseits wäre für Strontiumgo eine Frist von 250 Jahren notwendig, um die gleiche Reduktion zu erfahren. Die von atomischen Explosionen herrührende Aktivität wird innerhalb einer Woche auf I/500 reduziert [20a].

\section{Verdünnung}

Aktives Abwasser kann so sehr verdünnt werden, dass eine vorgeschriebene Toleranzgrenze unterschritten wird. In den USA. bestehen Verordnungen [38a], die vorschreiben, dass radioaktive Stoffe nur dann an das Kanalisationssystem abgegeben werden dürfen, wenn sie vorher auf eine Konzentration von weniger als O, I mc (Millicurie) verdünnt worden sind.

Eine besondere Art der Verdünnung ist die sogenannte chemische Verdïnnung (isotopic dilution). Zum Wasser werden stabile Isotope (carrier), die identische chemische Eigenschaften wie die entsprechenden radio- 
aktiven Substanzen aufweisen, zugegeben. Da Organismen nur eine beschränkte Menge von spezifischen Substanzen aufnehmen und nicht zwischen stabilen und instabilen Isotopen unterscheiden können, wird durch eine solche chemische Verdünnung die physiologische Schädigungsmöglichkeit reduziert. (Im Kantonsspital Zürich wird das anfallende Jod I3I vor der Abgabe an die Kanalisation chemisch verdünnt, indem pro Millicurie J I3 I I g inaktives Kaliumjodid zugegeben wird.)

\section{Entfernung des radioaktiven Materials aus dem Wasser}

Wasser, das mit radioaktiven Stoffen verunreinigt ist, kann nur durch Entfernung dieser Verunreinigungen gereinigt werden. Diese Art der Reinigung ist meistens umständlich, vor allem deshalb, weil je nach Art der radioaktiven Verunreinigungen verschiedene Methoden angewendet werden müssen. Doch ist es heute in den meisten Fällen möglich, zu einem befriedigenden Resultat zu gelangen. Allerdings ist das Problem damit noch nicht endgültig gelöst. Die dem Wasser entzogenen radioaktiven Substanzen müssen erst noch auf unschädliche Weise beseitigt werden.

\section{a) Destillation}

In einigen Fällen ist es notwendig, das aktive Wasser durch Destillation zu. reinigen. Das Verdampfungsverfahren ist kostspielig und kann nur für kleine Wassermengen angewendet werden. Die Kosten für die Verdampfung werden auf rund $30 \$ / \mathrm{m}^{3}$ berechnet [57], [33 a].

\section{b) Natürlicbe Fixierung}

Die Radioaktivität von Wasser kann - abgesehen vom Zerfall - durch einige natürliche Vorgänge reduziert werden. Vor allem können Bodenmaterialien und die in einem Fluss enthaltenen Schwebestoffe radioaktives Material fixieren. Diese Fixierung von aktiven Ionen an Bodenpartikel erfolgt vornehmlich durch Ionenaustauschadsorption, wobei radioaktive Ionen gegen eine äquivalente Anzahl von Ionen, welche ursprünglich an das Bodenmaterial gebunden waren, ausgetauscht werden. Das Ausmass dieser Austauschadsorption ist je nach Radioelement verschieden und ist hauptsächlich für Kationen von Bedeutung. Schwebestoffe können sowohl radioaktives Material adsorbieren als auch die Ausfällung radioaktiver Ionen begünstigen. Ein Beispiel zeigt Tabelle 5 .

Lebendes und totes Zellenmaterial kann die Aktivität des Wassers durch Adsorption und/oder Stoffwechselvorgänge reduzieren. Zudem sind 
Adsorption von Radioisotopen an lehmartige Schwebestoffe [25]

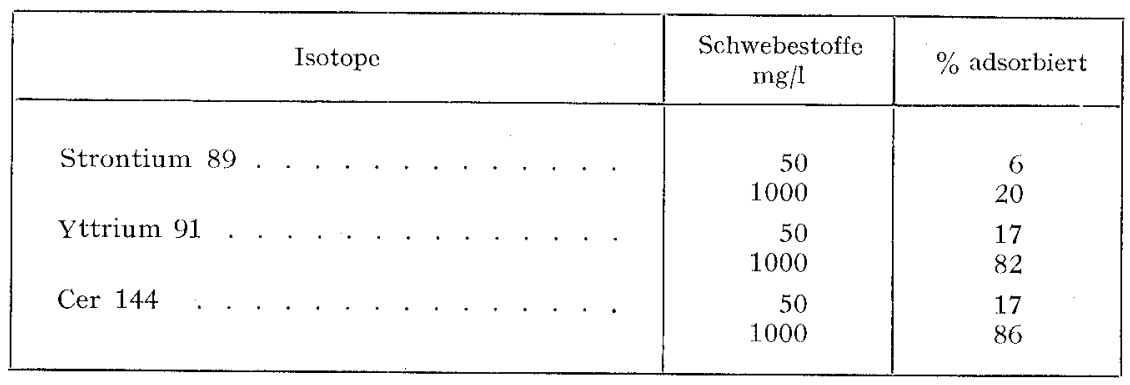

Wasserorganismen in der Lage, gewaltige Mengen radioaktiven Materials speichern. Nach PLACAC [25] weist I g (Trockengewicht) Algen, welche zu aus einem Cer I44 enthaltenden Wasser mit I, $8 \mathrm{I} . / \mathrm{min} / \mathrm{cm}^{3}$ (I. $\Longrightarrow$ Impulse) gezüchtet wurden, eine Aktivität von $3670000 \mathrm{I}$./min auf. Oberflächengewässer zeigen deshalb in bezug auf radioaktives Material ein gewisses Selbstreinigungsvermögen.

\section{c) Kontrollierte biologische Prozesse}

Die Stoffwechselprozesse von Wasserorganismen werden durch relativ grosse Konzentrationen radioaktiven Materials nicht beeinflusst. GRUNE und ELIASSEN [50] haben nachgewiesen, dass Ausmass und Geschwindigkeitskonstante des biochemischen Sauerstoffbedarfs durch Radio-Phosphor 32 bis hinauf zu ro mc/l nicht verändert wird. Es ist deshalb möglich, eine Reinigung durch Adsorption an Abwasserschlamm durchzuführen. RUCHHOFT [5I] hat die Verwendung des Belebtschlammverfahrens vorgeschlagen. Die Beseitigung des Schlammes stösst allerdings auf Schwierigkeiten (NEweLL [39]). Auch das Tropfkörperverfahren ist schon angewendet worden. Nach den Untersuchungen von RUCHHOFT und SETTER [52] können in Belebtschlammanlagen, die mit häuslichem Abwasser betrieben werden, durchschnittlich 36\% Phosphor 32 eliminiert werden. Der Reinigungseffekt des biologischen Verfahrens ist stark davon abhängig, ob das aktive Isotop chemisch verdünnt ist. So wird zum Beispiel (aktives) Jod I3 I, wenn es nicht mit Jod I27 vermischt ist, im Tropfkörper recht gut (mehr als 85\%) eliminiert (CARTER [49]). Andererseits beträgt die Elimination, wenn aktives und inaktives Jod (im Verhältnis I $\mathrm{g}$ KJ pro Millicurie J I3I) vermischt ist, in einer Belebtschlammanlage nur 10\% [52].

Oft enthalten radioaktive Abwässer synthetische Waschmittel (Deter- 
genzien) und Komplexbildner. Diese werden verwendet, um Räume, Betriebsgegenstände, Berufskleider, Messapparate usw., die mit aktivem Material in Berührung gekommen sind, wirkungsvoll zu reinigen. Dadurch werden alle Behandlungsmethoden zur Entfernung von radioaktiven Verunreinigungen (vor allem die Adsorptionsverfahren) erschwert. Durch Anwendung biologischer Reinigungsprozesse ist man oft in der Lage, die organischen Komplexbildner zu zerstören. Es ist deshalb gebräuchlich, solche Abwässer, vorgängig dem Adsorptionsverfahren, einer biologischen Reinigung zu unterzichen.

In diesem Zusammenhang mag darauf hingewiesen werden, dass in einer kommunalen biologischen Reinigungsanlage durch die Akkumulation von Radioisotopen im Schlamm die Verwertung des Abwasserschlammes für Düngezwecke erschwert werden kann.

\section{d) Adsorption}

Adsorptionsmethoden werden heute zum Teil auch zur Reinigung nichtaktiver industrieller Abwässer angewendet. Bei der Adsorption radioaktiver Isotope gelten die gleichen physikalisch-chemischen Gesetzmässigkeiten. Nur muss dabei allerdings berücksichtigt werden, dass die Gewichtskonzentrationen des radioaktiven Materials sehr gering sind. In Tabelle $\sigma$ sind als Beispiel für einige Isotope die entsprechenden Gewichts-

Tabelle 6

Gewicbtskonzentrationen einiger Isotope

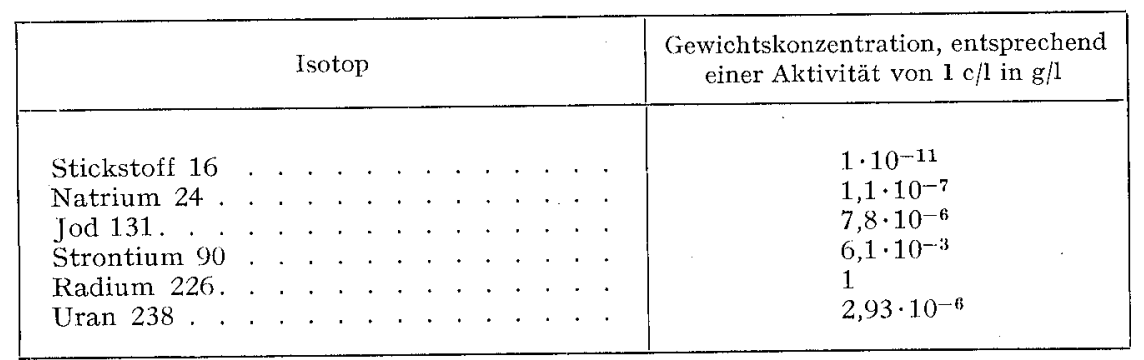

konzentrationen aufgeführt. So beträgt die höchste zulässige Konzentration von Jod I3 I im Trinkwasser $(0,3 \mathrm{mc} / \mathrm{l}) 2,34^{\cdot} \cdot \mathrm{IO}^{-12} \mathrm{~g} / 1 \mathrm{Jod}$, eine Menge, die mit den üblichen chemischen Methoden gar nicht nachweisbar ist. Oft müssen ausserordentliche Reinigungseffekte erzielt werden, um radioaktive Verunreinigungen in einem genügenden Ausmasse zu eliminieren. 
Vielfach erweist es sich als notwendig, Reinigungsfaktoren ${ }^{1}$ ) (Decontamination Factor) von Iooo und mehr zu erreichen.

Radioaktive Verunreinigungen können teilweise auch durch gewöhnliche Sandfiltration eliminiert werden. Dies ist besonders für die Wasserpersorgungspraxis von Bedeutung. Heute werden in der Schweiz I6\% des Trinkwassers einer Sandfiltration unterworfen ${ }^{2}$ ). BELL, THOMAS und RoseNTHAL [2I] haben die Adsorption von Aktivität durch Sandfilter in drei Wasserversorgungsanlagen von Neuengland bestimmt. Die Untersuchungen wurden in einem Zeitpunkt durchgeführt, in dem das Rohwasser, beeinflusst durch atomische Explosionen in Nevada, eine gut messbare Radioaktivität zeigte. Sie ergaben, dass in einer schnellen Sandfiltration etwa $2 \mathrm{I} \%$ der Aktivität entfernt wird. Diese Reinigungseffekte müssen darauf zurückgeführt werden, dass die Radioisotope im Oberflächenwasser bereits zu einem grossen Teil an Schwebestoffe, die durch den Sandfilter entfernt werden, adsorbiert sind.

Adsorption von Radioisotopen kann an natürlichen und synthetischen Ionenaustauschern erfolgen. Unter den natürlichen Austauschern werden vor allem kaolinitische oder montmorillonitische Tonmineralien verwendet. Diese zeigen eine geringere Kapazität als die synthetischen Austauscher, sind aber preislich günstiger. Mit synthetischen Harzen wird eine sehr wirksame Adsorption erreicht. Auch das Mischbettverfahren (Mischung von Kationen- und Anionenaustauschern) wird oft erfolgreich angewendet [54]. Das Ionenaustauschverfahren kann dann auf Schwierigkeiten stossen, wenn das zu reinigende Wasser grössere Salzkonzentrationen aufweist. Die mit Isotopen beladenen Austauscher müssen entweder beseitigt oder regeneriert werden. 1953 ist ein aussichtsreiches Verfahren patentiert worden [s3]. Radioaktive Kationen werden an montmorillonitische Tonmineralien adsorbiert. Das Tonmaterial wird dann bei rund $1000^{\circ} \mathrm{C}$ gebrannt. Dadurch werden die adsorbierten Ionen so fixiert, dass sie nachher nicht mehr ausgewaschen werden können. Das gebrauchte Tonmaterial kann deshalb bedenkenlos vergraben oder in einen See versenkt werden.

Koagulation. Bei diesem Verfahren werden im Wasser grobflockige Niederschläge von Eisenhydroxyd, Aluminiumhydroxyd, Calciumphosphat usw. erzeugt. Der Niederschlag reisst beim Absetzen die radioaktiven Verunreinigungen mit. Die kolloidchemischen Vorgänge beim Koagulationsverfahren sind heute zum 'Teil noch unabgeklärt. Immerhin kann man

1) Reinigungsfaktor $=$ Alstivität im Rohwasser: Aktivität im gereinigten Wasser.

2) Trinkwasserversorgungen in Gemeinden mit über 1000 Einwohnern. 
annehmen, dass die Entfernung von Radioisotopen durch Koagulation hauptsächlich auf folgenden Prinzipien beruht:

I. Es erfolgt reine Adsorption am grobflockigen Niederschlag, wobei das Ausmass der Adsorption von der Oberflächenwirkung des Niederschlages und von der Ladung der zu adsorbierenden Ionen abhängig ist (das höhergeladene Ion wird stärker adsorbiert).

2. Die radioaktiven Verunreinigungen werden bei der Niederschlagsbildung zum Teil zusammen mit dem Koagulationsmittel ausgefällt.

3. Kolloide Partikel können am grobflockigen Niederschlag adsorbiert oder bei der Fällung in diesen eingeschlossen werden (Okklusion).

Das Koagulationsverfahren bietet gegenüber anderen Reinigungsprozessen einige Vorteile:

I. Sowohl kleine als auch sehr grosse Abwasservolumina können behandelt werden.

2. Adsorption ist die wirkungsvollste Methode, um geringe Konzentrationen von Verunreinigungen aus dem Wasser zu entfernen.

3. Koagulationsoperationen können mit relativ bescheidenen und einfachen technischen Hilfsmitteln ausgeführt werden. Das Koagulationsverfahren ist deshalb oft wirtschaftlicher als andere Verfahren.

Eine gewöhnliche Koagulationsanlage besteht aus einem Koagulationsbecken, einem Absetzbecken und einem Sandfilter. Der abgesetzteSchlamm

Tabelle 7

Resultate pon Koagulationsexperimenten

\begin{tabular}{|c|c|c|c|c|}
\hline Isotop & Entfernung in \% & Koagulationsmittel & Bemerkungen & Literatur \\
\hline Jod 131 & weniger als 10 & Aluminiumsulfat & Laborexperiment & {$[56 a]$} \\
\hline Jod 131 & weniger als 1 & $\begin{array}{l}\text { Aluminiumsulfat } \\
+ \text { Absetzung } \\
+ \text { Sandfiltration }\end{array}$ & "Pilot»-Anlage & [46] \\
\hline Phosphor 32 & 98 und höher & Aluminiumsulfat & Laborexperiment & {$[56 \mathrm{a}]$} \\
\hline Phosphor 32 & 90 & Ferrisulfat & Laborexperiment & {$[23]$} \\
\hline Phosphor 32 & 85 & $\begin{array}{l}\text { Aluminiumsulfat } \\
+ \text { Absetzung } \\
+ \text { Sandfiltration }\end{array}$ & "Pilot"-Anlage & {$[46]$} \\
\hline Yttrium 91 & 45 & $\begin{array}{l}\text { Aluminiumsulfat } \\
+ \text { Natronlauge }\end{array}$ & Laborexperiment & {$[56 \mathrm{a}]$} \\
\hline Yttrium 91 & 99 & Calciumphosphat & Laborexperiment & {$[56]$} \\
\hline
\end{tabular}


kann mit Hilfe von Vakuumfiltern entwässert werden. RUCHнOFT [57] beschreibt eine Koagulationsanlage, die gebaut wurde, um geringe Konzentrationen von aktivem Plutonium zu entfernen. Diese Anlage zeigte Reinigungsfaktoren bis zu I000. Die Kosten der Reinigung betrugen aber immerhin rund $2 \$ / \mathrm{m}^{3}$. Dass auch das Koagulationsverfahren nicht in allen Fällen zu guten Resultaten führt, zeigt Tabelle 7 .

\section{c) Andere Metboden}

In speziellen Fällen gelingt es, Isotope an Metalle niederzuschlagen (Metallic Displacement) [37]. So können Yttrium, Ruthenium und einige andere Elemente relativ quantitativ mit Hilfe von Stahlwolle aus dem Wasser eliminiert werden. Ferner können noch folgende Methoden in Frage kommen: Lösungsextraktion, Elektrolytische Trennung, Elektrodialyse, Kristallisation.

\section{Die Beseitigung fester radioaktiver Abfälle}

Die anfallenden radioaktiven Abfälle (auch die bei der Reinigung dem Wasser entzogenen radioaktiven Substanzen) müssen beseitigt werden; dabei dürfen aber keine neuen Gefahrenherde entstehen. Das radioaktive Material muss unter streng kontrollierten Bedingungen aufbewahrt werden, wobei dessen Aktivität durch Zerfall abnehmen wird. Meist wird das aktive Material eingegraben. Dies muss mit der grössten Vorsicht geschehen, und die Stellen müssen dauernd überwacht werden. Es muss vor allem darauf geachtet werden, dass durch solche Ablagerungen Grundwasser nicht verunreinigt wird. Es wirkt sich günstig aus, dass manche Bodenmaterialien in der Lage sind, Radioisotopen durch Ionenaustausch festzuhalten. In den Vereinigten Staaten werden sogenannte «Radioaktivitätsfriedhöfe» gebaut, in denen der radioaktive Abfall gefahrenlos aufbewahrt werden kann. In den angelsächsischen Ländern werden radioaktive Abfallprodukte zum Teil ins Meer versenkt. Die damit zusammenhängenden Probleme werden von ScotT [43] und PoweLL [4I] diskutiert.

\section{METHODEN \\ ZUR MESSUNG DER RADIOAKTIVITÄT [57a]-[65]}

Radioaktive Strahlen sind für unsere Sinnesorgane direkt nicht wahrnehmbar; man benötigt zu deren Wahrnehmung technische Hilfsmittel, deren schematischer Aufbau hier ganz kurz erwähnt werden soll.

Alphateilchen erzeugen beim Aufprallen auf einen Fluoreszenzschirm kurzzeitige 
Lichtblitze (Scintillationen). Da solche Scintillationen relativ lichtschwach sind, werden sie mit Photozellen gemessen, wobei der schwache elektrische Strom verstärkt wird. Selbst Zeitintervalle von nur einem milliardsten Teil einer Sekunde zwischen dem Aufprallen zweier Teilchen lassen sich noch nachweisen. Scintillationszähler werden vor allem in sogenannten Koinzidenzanordnungen verwendet. Sie sprechen ebenfalls sehr gut auf Gammastrahlen an.

In der Wilson-Kammer erzeugt man ein mit Wasserdampf übcrsättigtes Gas, wobei cinc kleine Störung zur Kondensation von Nebeltröpfchen führt. Wenn nun ionisierende Strahlung in diese Kammer gelangt, so wirken die dabei entstehenden Ionen als Kondensationskeime, und es entsteht eine sichtbare Nebelspur.

In ähnlicher Weise können ionisicrende Strahlen einen pbotographischen Film beeinflussen. Jedes ionisierte Silberbromidpartikel bildet einen Keim, der beim Entwickeln der Platte wächst.

In der Wilson-Kammer und im photographischen Film wird die von der ionisierenden Strahlung hinterlassene Spur sichtbar gemacht. In der Ionisationskammer wird die Ladung der erzeugten Ionen gemessen. Eine solche Kammer besteht aus einem mit Gas gefüllten Gefäss, in dem sich zwei isolierte Elektroden befinden. An die Elektroden kann eine Spannung angelegt werden. Dic durch dic Strahlung erzeugten Ionen wandern $\mathrm{zu}$ den beiden Elektroden und erzeugen dadurch einen schwachen Strom, der mit einem Verstärker entsprechend vergrössert werden kann.

Die Verstärkung lässt sich vereinfachen oder umgehen bei der Verwendung des Zäblrobres (GEIGER-MüLLER). In der Achse eines Metallrohres ist ein dünner Draht isoliert befestigt. Das Rohr ist mit einer sehr verdünnten Gasmischung (zum Beispiel: Argon und Alkoholdampf; I/Io Atmosphäre) gefüllt. An das Metallirohr und den Draht wird cine hohe Spannung (1000 V und mehr) angelegt. Beim Auffallen ionisierender Strahlung auf den Zähler werden aus der Wand des Zählrohres Elektronen freigemacht. Infolge der angelegten Spannung fliegen die einzelnen Elektronen mit grosser Geschwindigkeit auf den Draht zu und erzeugen in der Drahtnähe eine Ionenlawine. Ein einzelnes Elektron kann bis zu einer Milliarde Ionen erzeugen. Diese riesige Zahl von Ionen lässt sich ohne Schwierigkeiten nachweisen.

Die Resultate werden als Impulse pro Minute registriert. Je nach der Art des Zählrohres und der Art der Strahlung kann aus der Anzahl der Impulse auf dic Radioaktivitätskonzentration geschlossen werden. Oft beträgt die «Ausbeute» (Verhältnis der Impulse zu Kernumwandlungen) nur 10\% oder weniger.

Kernumwandlungen finden nach statistischen Gesetzmässigkeiten statt. Bei der Messung von Radioaktivität müssen statistiscbe Gesichtspunkte befolgt werden. Das Resultat wird um so zuverlässiger, je mehr Impulse registriert werden. Bei der Messung von kleinen Aktivitäten muss die natürliche Grundstrahlung (hervorgerufen durch natürliche Radioaktivität in der Iuft und durch die kosmischen Strahlen) berücksichtigt werden. Präzise Messung von geringen Aktivitäten bedingt deshalb immer die Aufstellung ciner umfangreichen statistischen Analyse.

Die Messeinrichtung kann mit Präparaten bekannter Aktivität geeicht werden. Alle Messergebnisse bedürfen einer sorgfältigen Interpretation. Die Aktivität in Curie kann nur dann aus der Anzahl der Impulse berechnet werden, wenn bekannt ist, welche Isotope in der zu messenden Probe vorhanden sind und wie gross für jedes Isotop in der verwendeten Messeinrichtung die Ausbeute ist. Oft ist cs deshalb notwendig, vorgängig der Aktivitätsbestimmung cine Analyse oder Trennung durch- 
zuführen. Diese kann mit Hilfe chemischer 'Trennmethoden, durch Absorptionsmessungen oder durch Messung der Kernumwandlungsgeschwindigkeit (Zerfall) ausgefiihrt werden.

\section{ZUSAMMENFASSUNG \\ UND SCHLUSSFOLGERUNG}

Es ist damit zu rechnen, dass auch in der Schweiz in näherer Zukunft das Problem der Freihaltung des Trinkwassers vor radioaktiver Verunreinigung akut werden wird.

Die schädigende Wirkung radioaktiver Wasser wurde dargelegt und die technischen Möglichkeiten ihrer Reinigung beschrieben.

Es ist die Aufgabe der Wasserfachleute, die Reinigungsverfahren auszubauen und damit mitzuhelfen, die durch die moderne Atomphysik gegebene Entwicklung zu fördern.

\section{ANHANG}

\section{Definitionen und Einbeiten der Radioaktivität}

Unter Radioaktivität versteht man einen Prozess, bei dem instabile Atomkerne Energie in Form von Strahlung abgeben und dabei in stabile Kerne übergehen. Diese Umwandlung erfolgt unter Aussendung verschicdener Strahlung:

Alphastrablen bestehen aus Heliumkernen.

Betastrablung wird durch Elektronen hervorgerufen, welche sich annähernd mit Lichtgeschwindigkeit bewegen. Die Geschwindigkeit wird in Elektronenvolt gemessen.

Gammastrablen sind elektromagnetische Strahlen. Sie haben Lichtgeschwindigkeit. Sie können mit Röntgenstrahlen verglichen werden, nur haben sic eine kürzere Wellenlänge.

Isotope sind Elemente gleicher Ordnungszahl (Kernladungszahl), die sich im Atomgewicht unterscheiden. (Zur Kennzeichnung der Isotopen ist es üblich, das abgerundete Atomgewicht zusammen mit dem chemischen Symbol anzugeben, zum Beispiel: Jod I3I.)

Alle Strahlen verursachen eine Ionisierung des Materials, welches sie durchdringen. Alphastrahlen verursachen besonders starke, Gammastrahlen schwache Ionisation. Die Durchdringungskraft der Strahlen durch Material ist bei Gammastrahlen am grössten, bei Alphastrahlen am geringsten. Durch Kernumwandlung (Zerfall) nimmt die Aktivität eines radioaktiven Elementes mit der Zeit ab: $A=A_{0} e^{-\lambda i}$, wobei $A$ dic Aktivität zur Zeit $t, A_{0}$ die Aktivität zur Zeit $t={ }_{0}, \lambda$ die radioaktive Zerfallskonstante bedeuten. Die Halbmertszeit eines Elementes ist diejenige Zeit, die notwendig ist, um die ursprüngliche Aktivität auf die Hälfte zu reduzieren (Tabelle 2). 


\section{Einbeiten der Aktipität:}

Ein Curie (c) $=3,7 \cdot 10^{10}$ Kernumwandlungen pro Sekunde.

Ein Rutberford $=10^{6}$ Kernumwandlungen pro Sckunde.

\section{Finbeiten der Strablung:}

Ein Röntgen ( $\mathrm{r}$ ) ist diejenige Menge von Gammastrahlen (oder Röntgenstrahlen), die in $\mathrm{I} \mathrm{cm}^{3}$ trockener Luft im Normalzustand eine Anzahl Sekundärelektronen produziert, die in Luft Ionen mit einer Ladung von I elektrostatischer Einheit bilden. Die Röntgeneinheit ist kein ideales Mass für dic an biologische Objekte überführte Strahlungsmenge. Deshalb sind die rep- und die rem-Einheiten eingeführt worden. Ein rep (röntgen-equivalent-physical) ist die Strahlendosis, die im Gewebe eine Energieabsorption von $83 \mathrm{erg} / \mathrm{g}$ erzeugt.

lïn rem (röntgen-equivalent-man) ist diejenige Quantität einer beliebigen Strahlung, die im Gewebe denselben biologischen Effekt erzeugt wie I r Gammastrahlen. Strablungsdosis ist das Produkt aus Strahlungsintensität und Bestrahlungszeit.

Toleranzdosis ist die maximal zulässige Strahlendosis, die bei langdauernder Bestrahlung nicht überschritten werden darf, um gesundheitsschädliche Effekte mit grosser Wahrscheinlichkeit zu vermeiden.

\section{LITERATUR}

\section{Allgemeine Arbeiten}

[1] Gamov, G., Atomic Energy in Cosmic and Human Life (McMillan, 1946).

[2] Atomic Energy Commission, General Rules and Procedures Concerning Radioactipe Hazards, Branch Circular I-B (r947).

[3] US. Department of Health, Radiological Health Handbook (Cincinnati 1954).

[4] National Bureau of Standards, Safe Handling of Radioactipe Isotopes, Handbook 42 (I949).

[5] Weston, A. D., Radioactipity Enters Problems of Enpironmental Sanitation. (Mass. Public Health Department, I949).

[6] SchweITzer und WhitNey, Radioactipe Tracer Tecbnique (Van Nostrand I949).

[7] FRIEDLÄNDER und KENNEDY, Introduction to Radiocbemistry (Wiley, 1949).

[8] GUEST, G. H., Radio-Isotopes, Industrial Application (Toronto I95I).

[9] Zimen, K. E., Angemandte Radioaktivität (Springer-Verlag, Berlin I952).

\section{Strablungseffekte und Toleranzmerte}

[9a] Chalk River Conference (Canada 1950), in: US. Department of HEALTH, Radiological Health Handbook (Cincinnati I954).

[Io] CURTIS, H.J., The Biological Effects of Radiation, in: I. H. L.AWRENCE und H. Hamilton, Advances in Biological and Medical Physics, Bd. 2 (Academic Press, New York 1950). 
[it] Ingram, F. R., Radioactipe Protection Depices, J. Amer. Water Works Ass. 42, 537 (I950).

[12] International Commission on Radiological Protection (Kopenhagen I953).

[13] Lawrence, I. H., und H. Hamilton, Advances in Biological and Medical Physics (Academic Press, New York, Bd. I : I948; Bd. 2: I950).

[14] Lawrence, I. H., und C. A. ToBias, Advances in Biological and Medical Pbysics, Bd. 3 (I953).

[15] Minder, W., Utber biologische Wirkungen ionisierender Strablungen, Mitt. Lebensmitteluntersuch. Hyg. 46, 76 (1955).

[16] Morgan, K. Z., Tolerance Concentrations of Radioactive Substances, J. Phys. Coll. Chem. 51, 984 (I947).

[17] National Commission on Radiation Protection, Maximum Permissible Amounts of Radio-Isotopes in Human Body and Maximum Permissible Concentrations in Air and $W$ ater, Handbook 52 (Nat. Bureau of Standards, I953).

[18] Solomon, A. K., Kinetics of Biological Processes. Special Problems connected with the Use of Tracers, in: I. H. LAwrence und C. A. ToBIAS, Adpances in Biological and Medical Pbysics, Bd. 3 (1953).

[I9] SPOERL, R., The Lethal Effects of Radiation, Scientific American 1951, 185.

[20] STERN, C., Principles of Human Genetics (Freeman, San Franzisco 1950).

\section{Die Bedeutung der Radioaktivität in der Wasserpersorgung}

[20a] Atomic Energy Commission, Office of Civilian Defense, The Effect of Atomic Weapons, Kapitel 7 (1950).

[2I] Bell, C. G., H. A. Thomas jun. und B. L. Rosenthal, Passage of Nuclear Detonation Debris Through Municipal Treatment Plants, J. Amer. Water Works Ass. 46, 973 (I954).

[22] Damon, P. E., und P. K. Curoda, Artificial Radioactipity of Rainfall, Nucleonics $11,12,59$ (1953).

[23] Eliassen, R., Studies on Radio-Isotope Remopal by Water Treatment Processes, J. Amer. Water Works Ass. 43, 6r 5 (r9s3).

[24] Gorman, A. E., Radioactivity in Water Work Practice, J. Amer. Water Works Ass. 41, 1043 (I949).

[24a] Love, S. K., The Natural Radioactivity of Water, Ind. Eng. Chem. 43, I54I (195I).

[24b] Morris, J. R., und CARITT, Contamination of Water Supplies by Radioactive Substances, J. New Engl. Water Works Ass. 62, I (1948).

[25] Placac, O. R, Radioactivity Problem in Water Supplies (Atomic Energy Commission, T95 I).

[26] Rodger, W. A., Atomic Waste and Water 2uality, J. Amer. Water Works Ass. 42, 533 (1953).

[27] Staub, H., Neue Zürcher Z. 1954, Nr. 1099 (6. Mai).

[28] Straub, C. P., Removal of Radioactive Materials by Water Supply Facilities (Oak Ridge National Laboratory, I95 I).

[29] Thomas, H. A. Jun., et al., Radioactive Fallout in Surface Waters, J. Amer. Water Works Ass. 45, 562 (1953). 


\section{Beseitigung radioaktiver Stoffe}

[30] Eister, K. W., Treatment of Radioactipe Waste, Heating Ventil. 49, Nr. 9, 8I (I952).

[3I] GORMan, A. E. und W. Wolman, Nuclear Fission Operations and the Sanitary Engineer, Sew. Works J. 21, 637 (1949).

[32] Gorman, A. E., Disposal of $W$ aste from the Atomic Energy Industry, Waste Engr. $24,528(1953)$.

[33] Gorman, A. E., Disposal of Atomic Energy Industry Waste, Ind. Eng. Chem. 45, 2672. (I953).

[33 a] Hayner, I. H., Atomic Energy Industry, Ind. Eng. Chem. 44, 472 (I952).

[34] Hatch, L. P., Ultimate Disposal of Radioactipe Waste, American Scientist 41, 410 (I953).

[35] Hinsch, C. E., How Hanford Evaporates Fission Wastes, Chem. Engr. 60, I I (I953).

[36] Kaufman, W. J., und G. KLeIN, Radio-Isotope Remoral in Modern Waste Treatment, Amer. Soc. Civ. Engr. Proc. 79, 239 (I953).

[37] LARCY, W. J., Removal of Radioactive Material from $W^{Y}$ ater by Slurring with Pulperized Metal, J. Amer. Water Works Ass. 44, 824 (1952).

[38] Lauderdale, R. A., und A. H. Emmons, Decontamination of Small Volumes of Radioactipe $W^{\top}$ aters, Nucleonics 8 (2r. Mai I95 I).

[38a] National Bureau of STANDARDS, Handbook Nr. 49.

[39] Newell, J. F., und C. W. Christenson, Radioactive Waste Disposal, Sew. Ind. Waste 23, 86I (I95I).

[40] Placac, O., und Morton, Research and Disposal of Radioactive Waste, J. Amer. Water Works Ass. 42, I35 (1950).

[4I] Powell, C. L., und H. L. ANDrews, Disposal of Radioactipe Wastes at Sea, US. Publ. Health Rep. 67, 1214 (I952).

[42] Rodgers, W. A., und P. Fineman, $A$ Complete $W$ aste Disposal System for a Radiochemical Laboratory, Nucleonics 9, Nr. 6, 5I (I95I).

[43] ScOTT, K. G., Radioactipe Waste Disposal, Nucleonics 8 Nr. I., I 8 (I950).

[44] STRAUB, C. P., Observations on the Removal of Radioactive Materials from $W$ aste Solutions, Sew. Ind. Waste 23, I 88 (I95 I).

[45] Straub, C. P., Studies on Remopal of Radioactipe Wastes, J. Amer. Water Works Ass. 44,824 (I952).

[46] Straub, C. P., Industrial Wastes and Their Disposal, in: W. RudoLF, Industrial $W$ astes (Reinhold, New York 1953).

[17] Waste Disposal Symposium, Nucleonics 4 Nr. 3 (I949).

Biologische Reinigungsmethoden

[48] Biladeau, A. L., Trickling Filter Plant to Remove Radioactipity, Civil Engr. 23, 58

(I953).
[49] CARTER, M. W., Removal of Radioactive Isotope I 131 by the Use of Trickling Filters, Dissertation (Georgia Institute of Technology), und Sew. Ind. Waste 25, 560

(I953).
[50] GRUNE, W. N., und R. ELIASSEN, Studies on the Effect of Radioactipe Phosphorus on the Biocbemical Oxydation of Semage, Sew. Ind. Waste 23, I4I (195I). 
[5x] RuchHoft, C. (.., Possibility of Disposal of Radioactive Waste by Biological Treatment Metbods, Sew. Works J. 21, 877 (1949).

[52] Ruchioft, C. C., und L. R. SETTER, Application of Biological Methods in the Treatment of Radioactive Waste, Sew. Ind. Waste 25, 48 (I953).

\section{Ionenaustausch}

[53] GInell, W. S., Disposal of Radioactive Cations, US. Patent 2616847, Chem. Abstr. 47, I504 (I953).

[54] Ayres, J. A., Treatment of Radioactipe Waste by Ion Excbange, Ind. Eng. Chem. 43, I 526 (T95I).

\section{Koagulation}

[55] LACY, W. J., Remoding Radioactive Materials from Water by Coagulation, Water Sew. Works 100, 4IO (1953).

[56] LAUDERDALE, R. A., Removal of Radioactive Isotopes From Water by a Phosphat Coagulation and Floculation Process (Atomic Energy Commission, 1950).

[56a] Placac, O.. und Lyle, in: W. Rudolf, Industrial Wastes (Reinhold, New York 1953).

[57] Ruchhoft, C. C., A. E. Gorman und C. W. Christenson, Wastes Containing Radioactive Isotopes, Ind. Eng. Chem. 44, 545 (I952).

\section{Methoden zur Messung der Radioaktivität}

[57a] BALdingER, E., Zäbl- und Registriermethoden der Kernpbysik, Neue Zürcher Z. 1953, Nr. 2196 (29. September).

[58] CuRIS, L.F., Measurement of Radioactioity, Circular 476 (National Burcau of Standards, 1949).

[59] Evans, R. D., Fundamentals of Radioactioity and its Instrumentation, in: I. H. LAwRENCE und H. HAMrLTON, Advances in Biological and Medical Physics, Bd. 2 (Academic Press, New York, Bd. I: 1948; Bd. 2: 1950).

[60] FuURY, H., Elektronische Hilfsmittel als Scbutz gegen Atommaffen, Scientia Electrica 1955.

[6I] FreEdman, A. J., Low Lepel Counting Tecbniques, Nucleonics 10, Nr. 8, 57 (1952).

[62] Goldin, A. S., J. S. NADER und R. L. SeTtrer, Detectability of Low Ievel Radioactivity in Water, J. Amer. Water Works Ass. 45, 73 (I953).

[63] HAhN, R. B., und C. P. STRAUB, Determination of Radioactive Strontium and Barium in Water, J. Amer. Water Works Ass. 47, 335 (I955).

[64] HuRst, W. M., Monitoring of Liquids for Radioactivity.

[65] Task Group ReporT, Instrumentation and Methods of Testing Radioactipe Contamination in Water, J. Amer. Water Works Ass. 44, 583 (I952).

$$
\text { 6. Andere Arbeiten }
$$

[66] GüBELI, O., Zum Chemismus von Thermalmässern, Vjschr. Naturf. Ges. 98, I45 (I953).

[67] Melcher, D., Probleme radioaktiver Verseuchung pon Lebensmitteln, Mitt. Lebensmitteluntersuch. Hyg. 46, 68 (I955). 